F. DELl'ISOla AND K. HutTer

\title{
A Free Moving Boundary Problem for the Till Layer Below Large Ice Sheets
}

\section{Abstract}

We formulate a free moving boundary problem for the till (i.e., soil + water) layer that may form below glaciers or large ice sheets and is thought to be responsible for their catastrophic advance when the water content makes such layers slippery against shear deformations. We indicate how the FMBP is formulated, specialize it to steady plane flow and deduce an ordinary differential equation which describes the distribution of the solid's volume fraction across the layer. This differential equation is second order and gives rise to a singular perturbation solution procedure. This problem can be analysed under the assumption that the fluid viscosity is a monotonic function of the solid's volume fraction. However, in this paper we prove that by choosing a constant fluid viscosity and vanishing thermodynamic pressure the emerging solid volume fraction turns out to be physically meaningless.

\section{Introduction}

Glaciers and ice sheets which rest on the ground may respond critically to the local conditions of the materials of which this ground is composed. Typically one differentiates between hard and soft beds. By the latter one means that the ice rests on a thin layer (till layer) of a mixture of sediment and water which separates the ice from the rock bed. The horizontal motion of ice, which may slide along the upper interface, generates a (shear) deformation of this till layer while the ice overburden pressure as well as the melt water at the ice-till-layer interface in combination with the drainage of water and abrasion of till at the till-layer-rock-bed interface give rise to a vertical flow of water and sediment.

The dissipative mechanisms are the frictional heat due to the sliding of the till over the rock bed and of the ice over the upper interface, plus the dissipation provided by the stress power and the work done by the sediment-water-interaction force within the layer. The heat generated by them and the geothermal heat supplied from below are conducted and convected to the top interface to provide the heat necessary to melt ice that may become available once transformed into water to be pressed into the till layer to form the lubricand for its shear deformations.

A binary mixture model for the viscous behaviour of true density preserving constituents, granules and water, has been deduced from first principles of thermodynamics by Svendsen and Hutter (1995), and the general FMBP for the soil-water mixture bounded by the top and bottom interfaces as described above has been formulated 
by dell'Isola and Hutter $(1997,1998 \mathrm{a})$. Here we simply quote the relevant equations for the plane flow problem in which the thermal processes are ignored.

It is obvious from this description that the mechanical problem per se is already significant and that the water motion from above must play a significant role in the description of the distribution of the solid's volume fraction. The water that is pressed into the layer must loosen the granular matrix and will therefore provide the mechanism which reduces the resistance of the layer to shear deformations. It shall be demonstrated in this paper that plane flow is described by a FMBP. By specializing this problem to steady conditions, a two-point boundary value problem can be deduced for the solid's volume fraction with fixed boundaries. Dell'Isola and Hutter (1998b) have shown that this boundary value problem is stiff, and they have analysed it for nonvanishing thermodynamic pressure $\beta_{s}$ when the fluid viscosity is constant. In this paper we prove that with vanishing thermodynamic pressure and constant fluid viscosity the emerging analytical profiles of solid volume fraction are physically meaningless.

\section{Governing equations}

Let $O x y z$ be a Cartesian coordinate system with origin $O$, horizontal axes $(x, y)$ and vertical axis $z$. Consider a layer of a saturated binary mixture of granules and a fluid bounded by $z=f_{b}(x, y, t)$ and $z=h(x, y, t)$ and let $f_{b}<h$. Assume the material within this layer to be subject to plane deformations such that $\partial(\cdot) / \partial y=0$ for any field variable, and suppose that all fields obey the condition $\partial(\cdot) / \partial x=0$ except the saturation pressure $p(x, t)$. Consider the following list of variables and parameters:

\begin{tabular}{|c|c|}
\hline$\nu$ & solid volume fraction, \\
\hline$u_{s}, u_{f}$ & $\begin{array}{l}\text { horizontal velocity components of the solid and } \\
\text { fluid, respectively, }\end{array}$ \\
\hline$w_{s}, w_{f}$ & $\begin{array}{l}\text { vertical velocity components of the solid } \\
\text { and fluid, respectively, }\end{array}$ \\
\hline & saturation pressure, \\
\hline$\beta_{s}(\nu)$ & themodynamic pressure, \\
\hline$\hat{\rho}_{f}, \hat{\rho}_{s}$ & true fluid and sediment mass densities, \\
\hline $\begin{array}{l}\mu_{f}, \mu_{s} \\
\tilde{\alpha}=: \hat{\rho}_{f} g / K\end{array}$ & (apparent) fluid and solid viscosities, \\
\hline$a:=\frac{\hat{\rho}_{f}}{\hat{\rho}_{s}}$ & $\xi_{s}:=\frac{\rho_{s}}{\rho}=\frac{\nu \hat{\rho}_{s}}{\nu \hat{\rho}_{s}+(1-\nu) \hat{\rho}_{f}}=\frac{\nu}{\nu+(1-\nu) a}$ \\
\hline $\begin{array}{l}g \\
K\end{array}$ & $\begin{array}{l}\text { gravity constant, } \\
\text { soil permeability. }\end{array}$ \\
\hline
\end{tabular}

Dell'Isola and Hutter (1998b) explain that plane deformation of the sediment water 
mixture is described by the following set of partial differential equations:

$$
\begin{aligned}
& \frac{\partial \nu}{\partial t}+\left(\nu w_{s}\right)^{\prime}=0, \\
& -\frac{\partial \nu}{\partial t}+\left((1-\nu) w_{f}\right)^{\prime}=0, \\
& \left(-\nu \beta_{s}-\nu p+\mu_{s} w_{s}^{\prime}\right)^{\prime}+\left(p+\left(1-\xi_{s}\right) \beta_{s}\right) \nu^{\prime}+ \\
& -\nu(1-\nu) \tilde{\alpha}\left(w_{s}-w_{f}\right)=0 \\
& \left(-(1-\nu) p+\frac{4}{3} \mu_{f} w_{f}^{\prime}\right)^{\prime}-\left(p+\left(1-\xi_{s}\right) \beta_{s}\right) \nu^{\prime}+ \\
& +\nu(1-\nu) \tilde{\alpha}\left(w_{s}-w_{f}\right)=0 \\
& -\nu \frac{\partial p}{\partial x}+\left(\mu_{s} u_{s}^{\prime}\right)^{\prime}-\nu(1-\nu) \tilde{\alpha}\left(u_{s}-u_{f}\right)=0, \\
& -(1-\nu) \frac{\partial p}{\partial x}+\left(\mu_{f} u_{f}^{\prime}\right)^{\prime}+\nu(1-\nu) \tilde{\alpha}\left(u_{s}-u_{f}\right)=0, \\
& \quad \text { in } z \in\left(f_{b}, h\right), t>0
\end{aligned}
$$

in which the prime denotes differentiation with respect to $z$, and $\beta_{s}$ and $\mu_{f}$ are prescribed functions of $\nu$,

$$
\left\{\begin{array}{l}
\beta_{s}=\beta_{0}+\check{\beta}_{s}(\nu), \quad \check{\beta}_{s}(0)=0, \\
\mu_{f}=\mu_{f}(\nu),
\end{array}\right.
$$

to be specified below. Physics suggests $\mu_{f}$ to monotonically increase with $\nu$. Moreover, $\beta_{s}$ must satisfy the inequality

$$
\frac{d \beta_{s}}{d \nu}-\beta_{s} \frac{1}{\nu+b} \geq 0, \quad b=\frac{a}{1-a}
$$

Note that equations $(2)_{1-4}$ are uncoupled from $(2)_{5,6} ;$ a similar decoupling also applies when abrasion is ignored as seen in dell'Isola and Hutter (1998b). We thus ignore $(2)_{5,6}$, only deal with $(2)_{1-4}$ and quote the following boundary conditions from the aforementioned paper:

- At the top interface $z=h(t)$

$$
\begin{aligned}
& w_{f}(1-\nu)=-V_{f 0} \\
& \frac{\partial h}{\partial t}=w_{s} \\
& -\alpha \nu^{\prime} p_{i}=-\nu\left(\beta_{s}+p\right)+\mu_{s} w_{s}^{\prime}, \\
& -\left(1-\alpha \nu^{\prime}\right) p_{i}=-(1-\nu) p+\frac{4}{3} \mu_{f} w_{f}^{\prime} .
\end{aligned}
$$

in which $V_{f 0}$ is the absolute value of the volume flow per unit area of water from the top into the layer and $\alpha \in(0,1)$, while $l \simeq 2 / 3$.

- At the bottom surface $z=f_{b}(t)$

$$
\begin{aligned}
& \frac{\partial f_{b}}{\partial t}=-w_{b}=-\frac{\mathcal{M}_{a}^{b}}{\rho_{r}}, \\
& (1-\nu)\left(w_{f}-w_{b}\right)=\frac{\mathcal{M}_{f}^{b}}{\hat{\rho}_{f}}=-\frac{m_{f}^{b}}{\hat{\rho}_{f}} \sigma_{f}^{4}, \\
& \nu\left(w_{s}-w_{b}\right)=-\frac{\mathcal{M}_{d}^{b}}{\hat{\rho}_{s}}
\end{aligned}
$$


with

$$
\sigma_{f}:=-(1-\nu) p+\frac{4}{3} \mu_{f} w_{f}^{\prime},
$$

in which $\mathcal{M}_{s}^{b} / \rho_{r}$ is the abrasion rate, henceforth set to be zero, while $\mathcal{M}_{f}^{b} / \hat{\rho}_{f}$ is the drainage rate of the water into the rock bed which we assume to be proportional to the fourth power of $\sigma_{f}$, the fluid pressure normal to the bottom surface.

Moreover, in dell'Isola and Hutter (1998b) it is shown that the above equations, complemented by initial conditions for $\nu, f_{b}$ and $h$, are likely a well-posed initial value FMBP for the fields $p, \nu, w_{s}$ and $w_{f}$, provided $V_{f 0}$, the incumbent pressure and the abrasion rate function are a priori known.

\section{Steady-state conditions with vanishing abrasion.}

Let abrasion be zero and $V_{f 0}$ as well as $p_{0}$ be time independent. Then, as show in dell'Isola and Hutter (1998b), the above FMBP transforms into the following twopoint boundary-value problem for the solids volume fraction $\nu$ expressed in the new variable

$$
\begin{gathered}
y:=\frac{1}{1-\nu}, \quad y \in[1, \infty] \\
-\left(\tilde{\mu}_{f} \delta_{f}\right) y^{\prime \prime}+\left(h(y)-\tilde{\mu}_{f}^{\prime} \delta_{f}\right) y^{\prime}+\eta_{f}=0, \quad \tilde{z} \in(0,1) \\
-\delta_{f} \tilde{\mu}_{f} y^{\prime}+\frac{y-1}{y^{2}} \tilde{\beta}_{s}+\frac{1}{y} \frac{p_{i}}{\left[\beta_{1}\right]}-\mathcal{F}=0, \quad \text { at } \tilde{z}=0, \\
-\delta_{f} \tilde{\mu}_{f} y^{\prime}+\frac{y-1}{y^{2}} \tilde{\beta}_{s}+\left(\frac{y-1}{y}-\alpha\left(\frac{y-1}{y}\right)^{\prime}\right) \frac{p_{i}}{\left[\beta_{1}\right]}=0, \quad \text { at } \tilde{z}=1,
\end{gathered}
$$

in which

$$
\left\{\begin{array}{c}
\eta_{f}:=\tilde{\alpha} \frac{\left[V_{f 0}\right][H]}{\left[\beta_{1}\right]}=O\left(10^{-7}-10^{-4}\right), \\
h(y(\nu))=(1-\nu)^{3}\left(\tilde{\beta}_{s} \frac{1}{\nu+b}+\frac{d}{d \nu} \tilde{\beta}_{s}\right), \\
\delta_{f}:=\frac{4}{3} \frac{\left[\mu_{f}\right]\left[V_{f 0}\right]}{[H]\left[\beta_{1}\right]}=O\left(10^{-20}-10^{-17}\right), \\
\mathcal{F}:=\left(\frac{V_{f o} \hat{\rho}_{f}}{m_{f}^{b}}\right)^{1 / 4} \frac{1}{\left[\beta_{1}\right]} \simeq O\left(10^{-1}-10^{0}\right),
\end{array}\right.
$$

and $[\cdot]$ - quantities are scales for the variables in brackets, $[H]$ being the constant thickness of the layer. Furthermore, $z=[H] \tilde{z}$ and primes now denote differentiations with respect to $\tilde{z}:(\cdot)^{\prime}=d(\cdot) / d \tilde{z}$. Note that the differential equation $(9)_{1}$ has the following properties: 
when $\tilde{\beta}_{s} \equiv 0$, i.e., when the thermodynamic pressure is neglected, then (9) reduces to the differential equation

$$
\left(\tilde{\mu}_{f}(y) y^{\prime}\right)^{\prime}=\frac{3}{4} \tilde{\alpha} \frac{[H]^{2}}{\left[\mu_{f}\right]}=: \frac{1}{\varepsilon} \quad\left(\simeq 10^{15}\right)
$$

the integration of which, subject to the boundary conditions

$$
y(0)=y_{0}, \quad y(1)=y_{h}, \text { with } y_{h}<y_{0}
$$

yields

$$
M\left(y ; y_{h}\right)=\frac{1}{2 \varepsilon}\left(\tilde{z}^{2}-\tilde{z}\right)+M\left(y_{0} ; y_{h}\right)(1-\tilde{z})=: \tilde{M}(\tilde{z})
$$

with

$$
M\left(y ; y_{h}\right):=\int_{y_{h}}^{y} \tilde{\mu}_{f}(\bar{y}) d \bar{y} .
$$

Plotting $\tilde{M}(\tilde{z})$ in the interval $0 \leq \tilde{z} \leq 1$ gives the parabola with values zero at $\tilde{z}=1$ and $M\left(y_{0} ; y_{h}\right)$ at $\tilde{z}=0$, and the minimum $\tilde{M}\left(\tilde{z}^{*}\right)=-\frac{1}{2 \varepsilon}\left(\frac{1}{2}-\varepsilon M\left(y_{0} ; y_{h}\right)\right)^{2}$ at $\tilde{z}^{*}=\left[\frac{1}{2}+\varepsilon M\left(y_{0} ; y_{h}\right)\right]$. Whether or not $\tilde{z}^{*}$ lies in $[0,1]$ depends upon the functional dependence of $\tilde{\mu}_{f}$ on $y$. For constant $\tilde{\mu}_{f}=1, \tilde{z}^{*}=\left[\frac{1}{2}+\varepsilon\left(y_{0}-y_{h}\right)\right]$ and (13) becomes

$$
y(\tilde{z})=\frac{1}{2 \varepsilon}\left(\tilde{z}^{2}-\tilde{z}\right)-\left(y_{0}-y_{h}\right) \tilde{z}+y_{0} .
$$

The function $y(\tilde{z})$ has values $y_{0}$ and $y_{h}$ at $\tilde{z}=0$ and at $\tilde{z}=1$, respectively, and decreases into the interval $\tilde{z} \in[0,1]$ to reach the negative minimum $y_{\min }=y_{h}-$ $\frac{1}{2 \varepsilon}\left(1-\varepsilon\left(y_{0}-y_{h}\right)\right)^{2}$ at $\tilde{z}=\tilde{z}^{*}$. Correspondingly, the function

$$
\nu(\tilde{z})=1-\frac{1}{y(\tilde{z})}
$$

shows in $\tilde{z} \in[0,1]$ three branches separated by two singularities when $y\left(\tilde{z}_{s}\right)=0$. The two roots $\tilde{z}_{s}^{1}, \tilde{z}_{s}^{2}$ are in the neighbourhood of $\tilde{z}=0$ and $\tilde{z}=1$, respectively, and define two boundary layers with thicknesses of order $\varepsilon$. The properties of $\nu(\tilde{z})$ are

- For $\tilde{z} \in\left[0, \tilde{z}_{s}^{1}\right], \nu(\tilde{z})$ starts with a positive value at $\tilde{z}=0$ and approaches $\mp \infty$ as $\tilde{z} \rightarrow \tilde{z}_{s}^{1 \mp}$.

- For $\tilde{z} \in\left[\tilde{z}_{s}^{2}, 1\right], \nu(\bar{z})$ is positive at $\bar{z}=1$ and decreases with decreasing $\tilde{z}$, approaching $\mp \infty$ as $\bar{z} \rightarrow \tilde{z}_{s}^{2 \pm}$.

- For $\tilde{z} \in\left[\tilde{z}_{s}^{1}, \tilde{z}_{s}^{2}\right], \nu(\bar{z})$ is larger than unity and singular, i.e., $\nu(\tilde{z})$ tends to $+\infty$ for $\tilde{z} \rightarrow \tilde{z}_{s}^{1+}$ and $\tilde{z} \rightarrow \tilde{z}_{s}^{2-}$, respectively. 
Such behaviour is physically unacceptable not only because $\nu(\tilde{z})$ is larger than 1 in the middle of the layer but equally so because it also attains twice an infinite modulus within the layer.

This result proves that $\tilde{\mu}_{f}$ cannot be constant, or the thermodynamic pressure must be assumed to be nonvanishing, or both. This is demonstrated elsewhere.

\section{Acknowledgments}

We thank Kurt Frischmuth for intriguing discussions on viscosity. K.H. acknowledges financial|support by the A.v. Humboldt foundation and the Max Planck Society through its Max Planck Prize.

\section{References}

[1] Dell'Isola F. AND Hutter K. 1997 Continuum mechanical modelling of the dissipative processes in the sediment-water layer below glaciers C. R. de l'Acad. Sci. Paris, t. 325, Ser IIb (1997), 449 - 456.

[2] Dell'Isola F. AND HutTer K. 1998a What are the dominant thermomechanical processes in the basal sediment layer of large ice sheets? Proc. $R$. Soc. London, A 454 (1998), 1169 - 1196.

[3] Dell'Isola F. AND Hutter K. 1998b A qualitative analysis of the dynamics of a sheared and pressurized layer of saturated soil Proc. R. Soc. London in press.

[4] Svendsen B. AND Hutter K. 1995 On the thermodynamics of a mixture of isotropic materials with constraints Int. J. Engng. Sci. 33, 14, p. 2021-2054.

Francesco dell'Isola

Dipartimento di Ingegneria Strutturale e Geotecnica

Universita' di Roma LA SAPIENZA

Via Eudossiana 18, I-00184 Roma

Kolumban Hutter

Institut für Mechanik, Technische Universität Darmstadt, D-64289 Darmstadt, Deutschland 